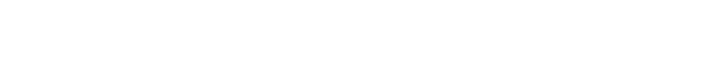

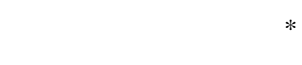

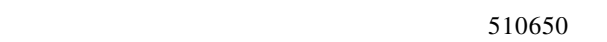

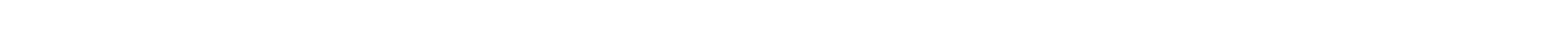

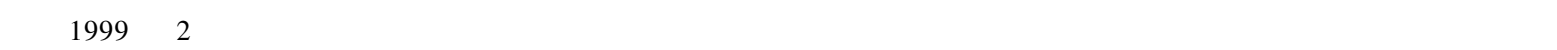

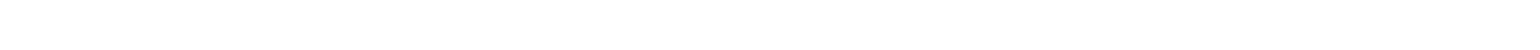

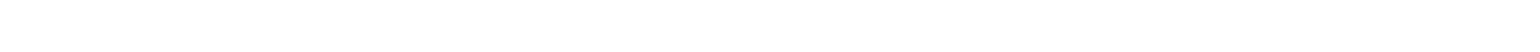

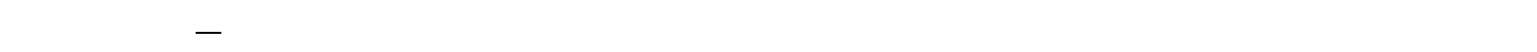

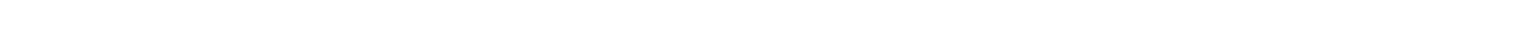

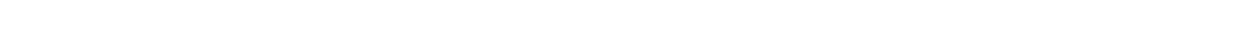

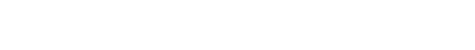

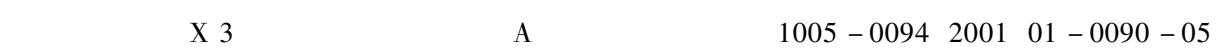

\section{Management strategies for invasive species in the United States}

LIANG Xiao-DongfłE Wan-Hui

South China Institute of Botanyf-Chinese Academy of sciences $\mathbf{f - C u a n g z h o u ~} 510650$

\begin{abstract}
The invasion of alien species is a major factor leading to the biodiversity crisis. Biological invasion is a major cause of biodiversity loss in the United States. The U. S. government is consistently paying more attention to invasive species now. On February $3 £-1999 f-$ President Clinton signed an executive ordef" EO $@$ to coordinate a federal strategy to address the growing environmental and economic threat from invasive plants and animals that are not native to the United States. The Presidenti $\overline{\mathrm{s}}$ order directs federal agencies to use their authority to prevent the introduction of invasive species and to restore native species. The EO directs establishment of an Invasive Species Council and prescribes the duties of the Council and federal agencies. The National Strategy for Invasive Plant Managementf-supported by the U.S. Department of Agriculture and other departments $£$ proposes three national goals $£-$ prevention and control of invasive species $\mathbf{i}^{-}$and restoration. This National Strategy provides three mechanisms $\mathfrak{}^{-}-{ }_{\text {partner- }}$ shipsfeducationfand research for different groups and individuals. It requires a high level of cooperation and collaboration among them. Aggressive federal actions are already underwayfincluding measures to prevent entry of invasive speciesf control and eradication of invasive speciesfoutreach and education for the general publicfand an increase in the budget for each fiscal year.
\end{abstract}

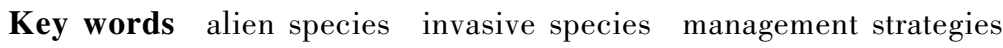

\section{1 Ò̃र̃̂}

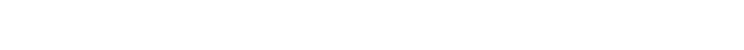

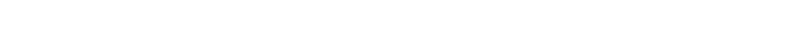

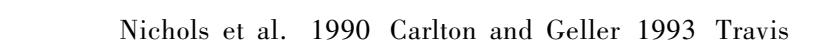

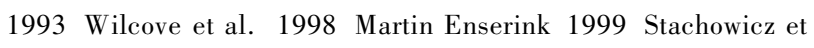

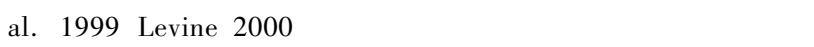

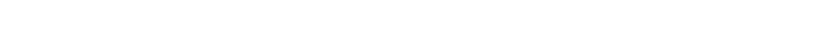

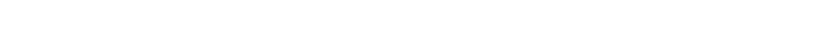

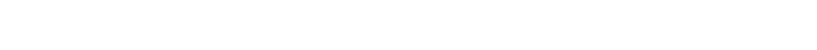

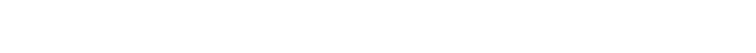




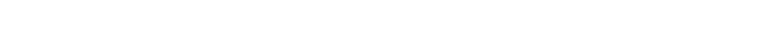

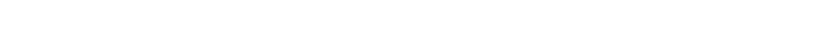

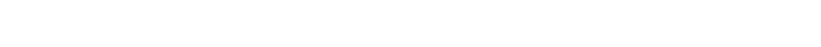

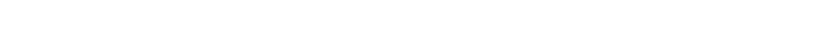

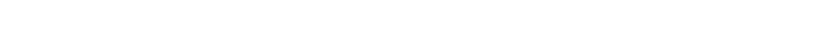

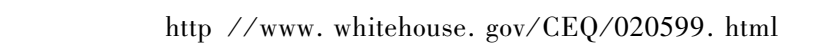

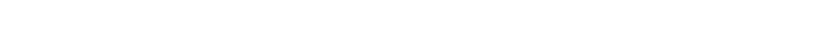

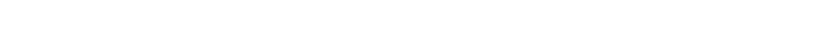

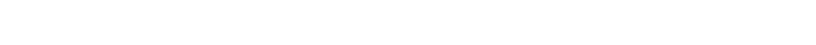

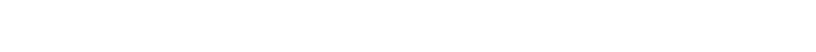

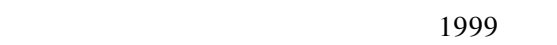

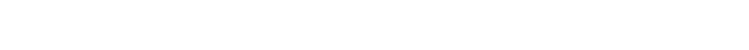

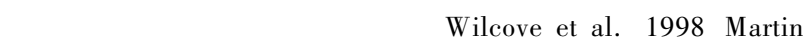

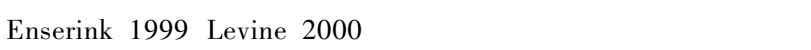
È x’ İ $\rightarrow$

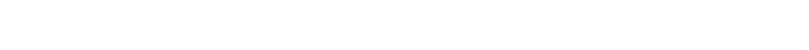

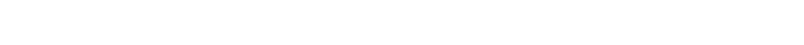

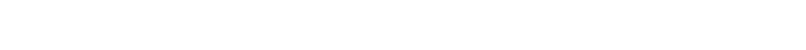

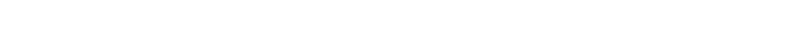

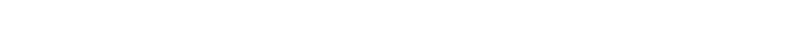

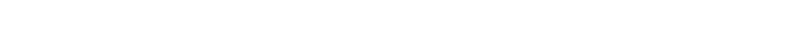

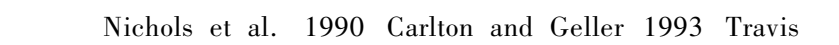

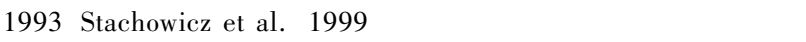

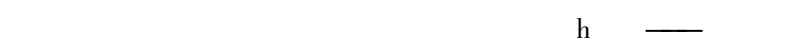

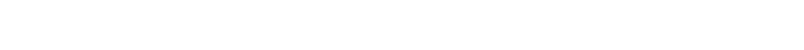

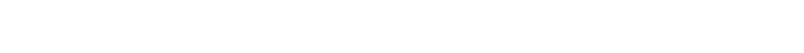

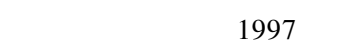

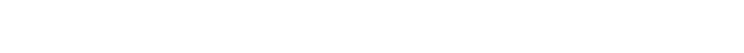
ÖßÒ>£每有 'î

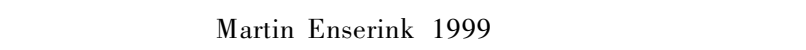
Îi

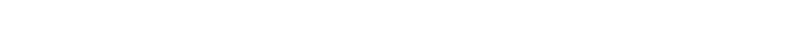

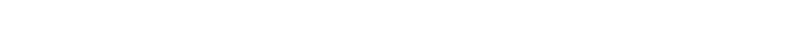

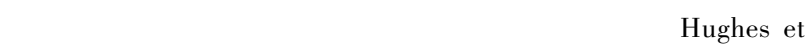

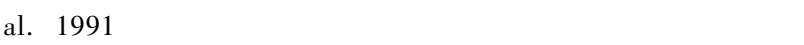

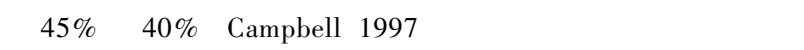

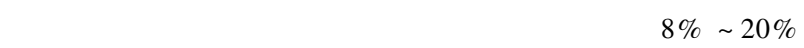

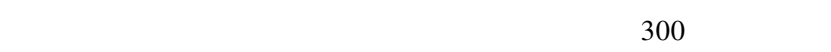

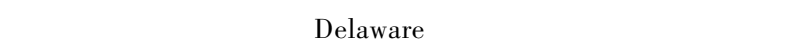

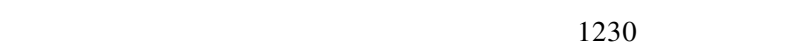
Èç $\mathrm{f}$

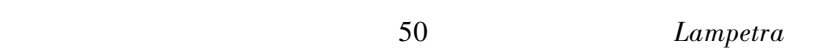

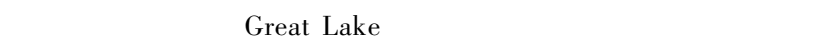

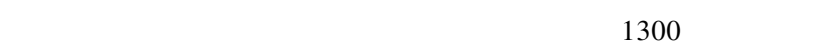

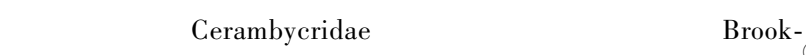

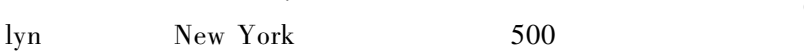

Îi Ã ÄÂề̆

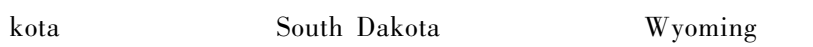

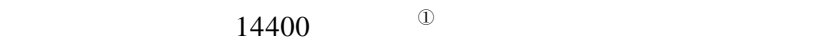

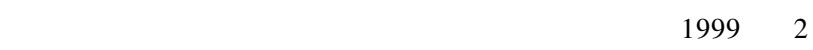

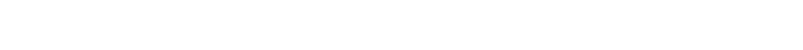

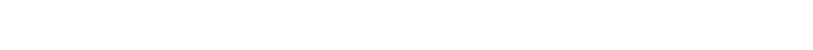

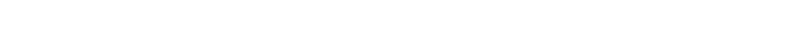

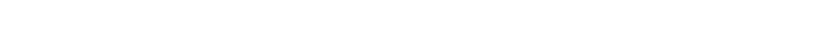

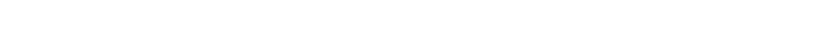

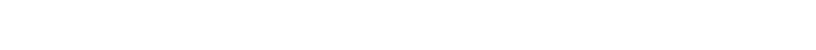

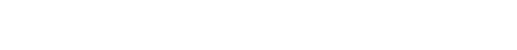

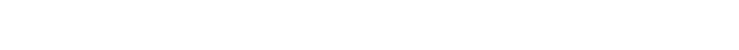

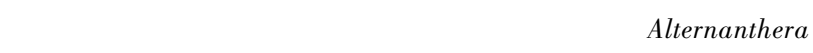

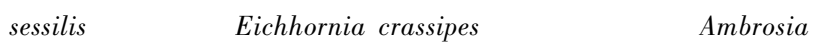

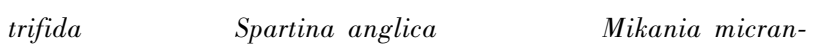
tha

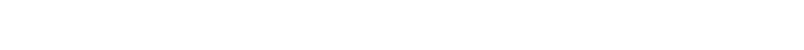

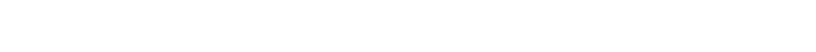

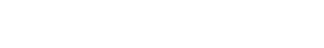

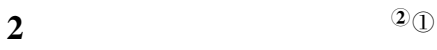

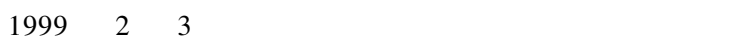

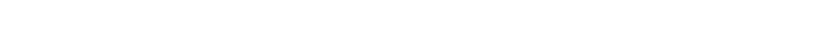

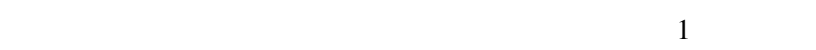

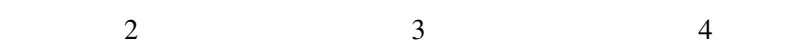

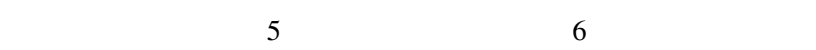
Ài if

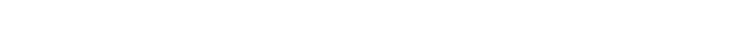

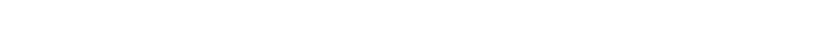

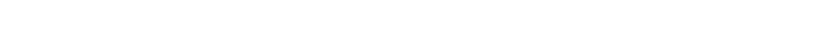

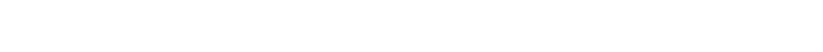

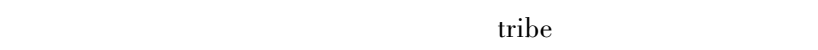

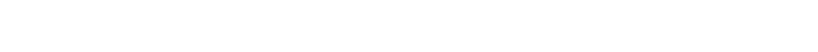

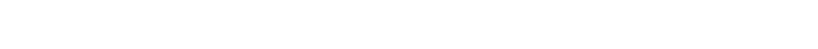

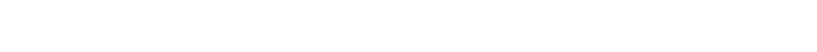

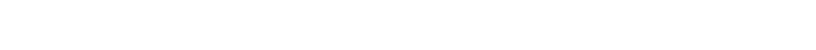

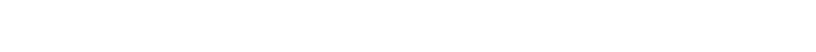

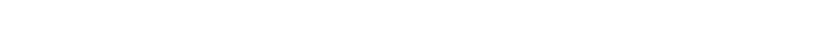
ÉÓi

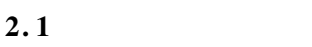

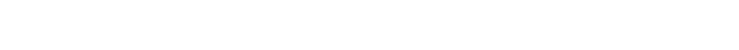

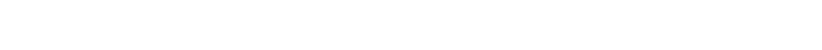

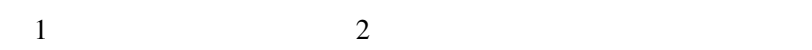

×ÊÄ À Ố foll http fq / www. whitehouse. gov/CEQ/020599. html (2) http $f$ / refuges. fws. gov/FICMNEWFiles/eo. html 


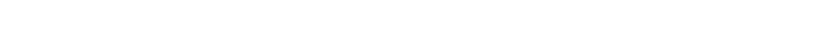

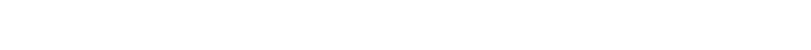

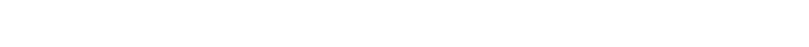

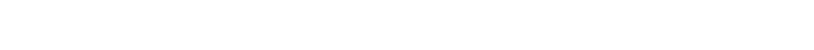

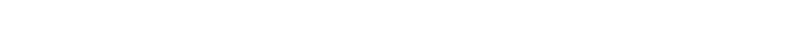

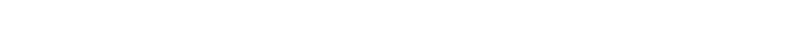

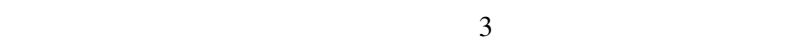

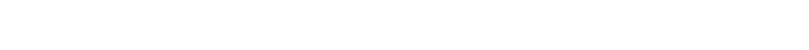

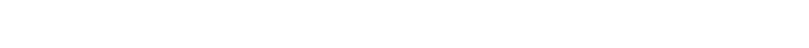

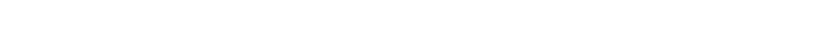

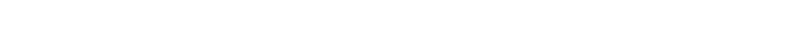

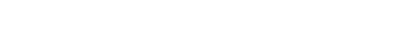

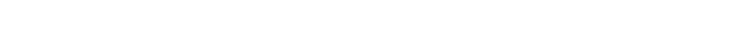

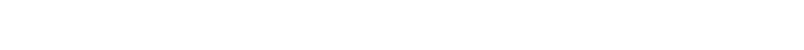
Ò)Ö̂̂f

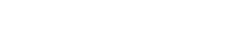

\section{2 À ÊÂ»ánÄメé}

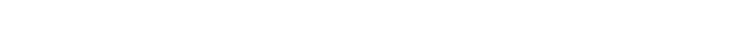

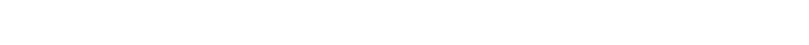

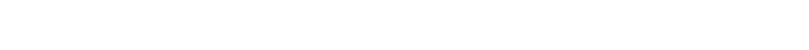

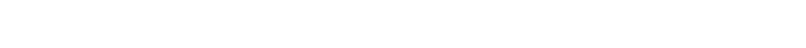

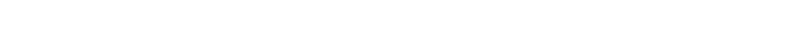
$\mu \ddot{A ̈}^{\circ} \grave{i}$ ÊÂA

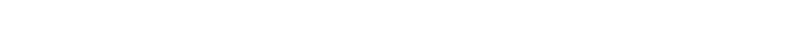

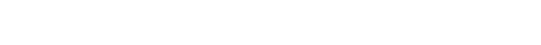

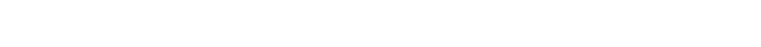

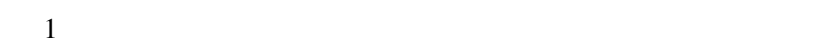

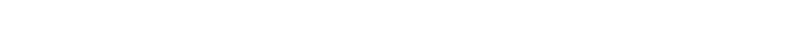

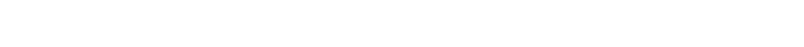

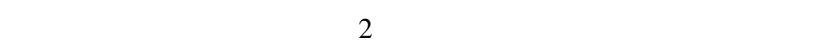

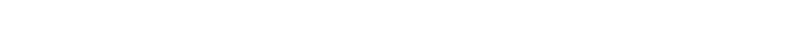

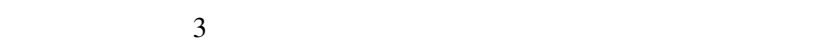

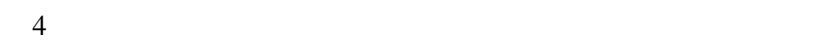

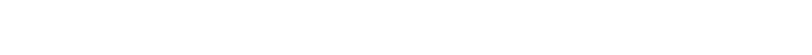

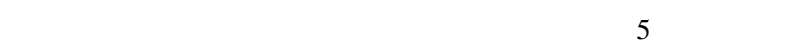

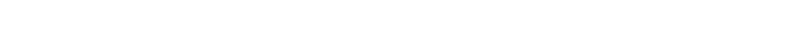

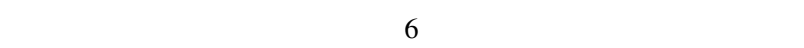

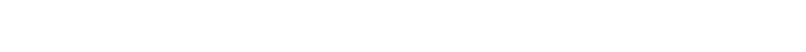

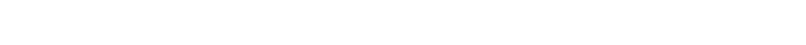

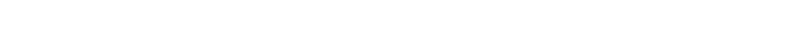

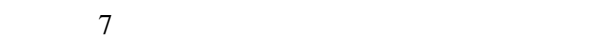

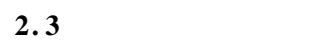

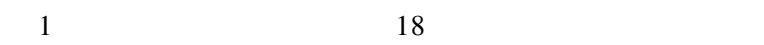

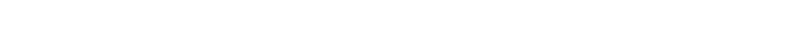

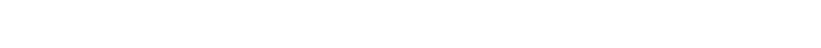

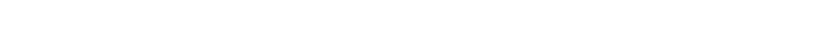

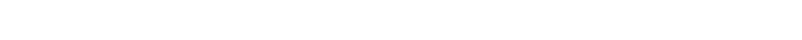

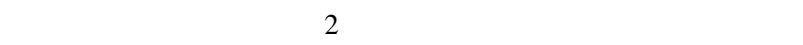

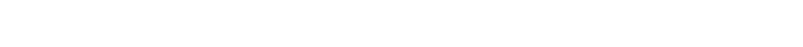

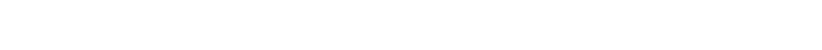

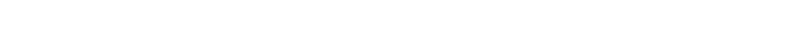

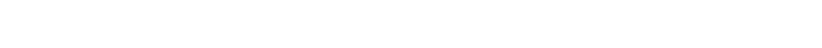

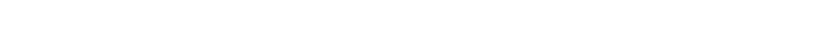

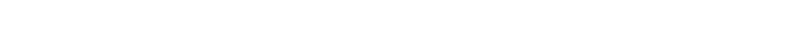

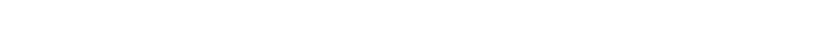

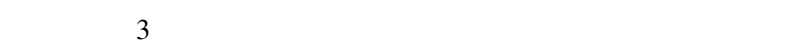

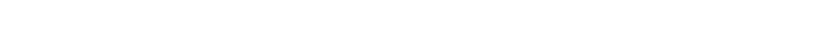

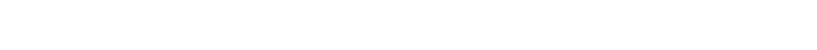

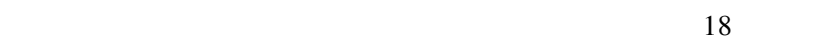

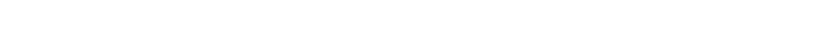

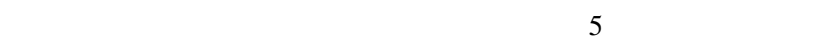

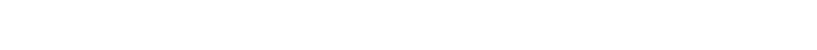

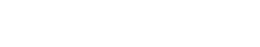

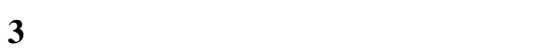

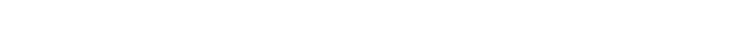

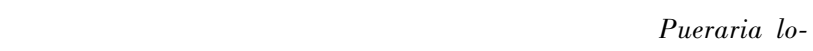

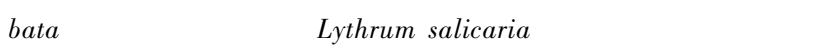

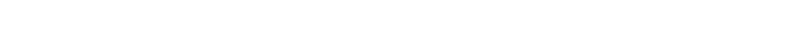

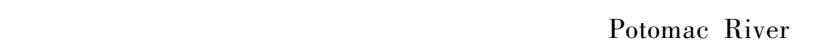

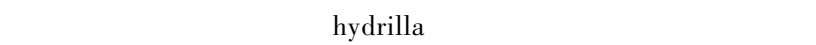

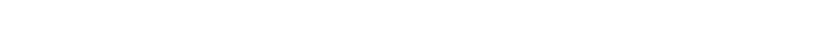

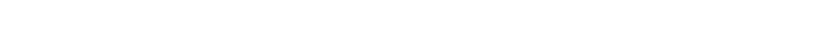

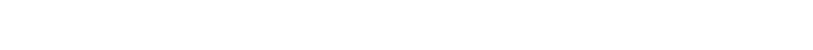

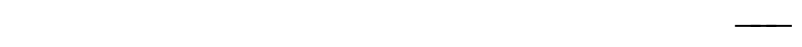

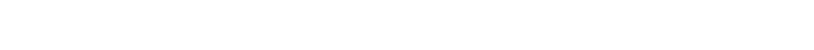

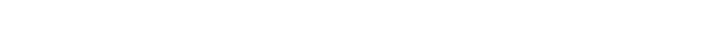

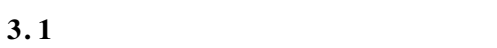

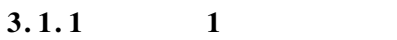

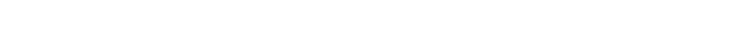

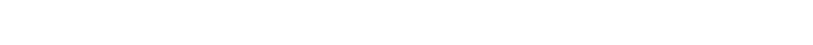

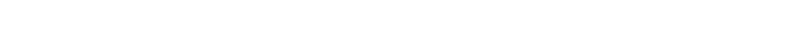

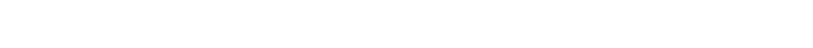

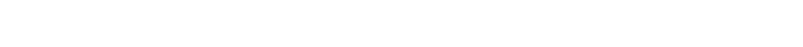

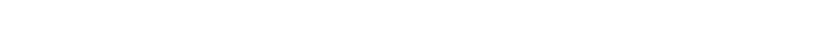
'óÅú

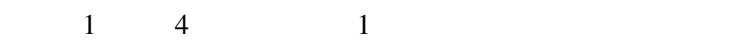

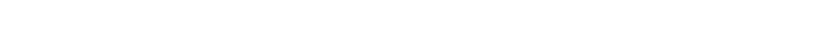

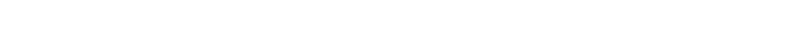

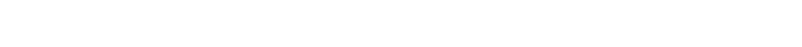

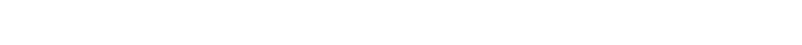

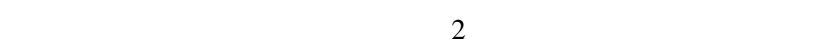




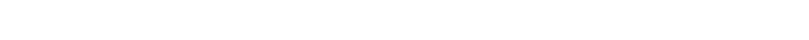

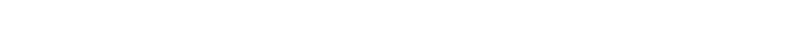

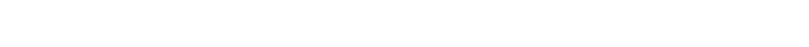

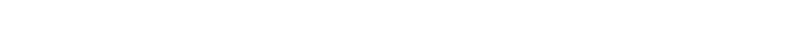

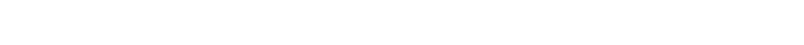

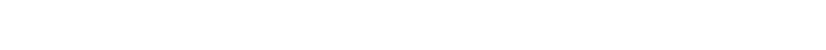

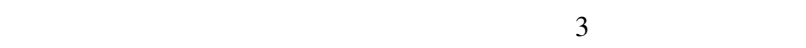

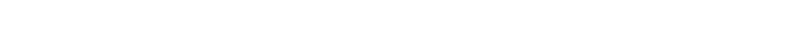

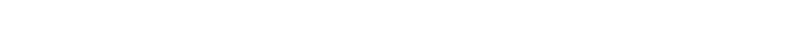

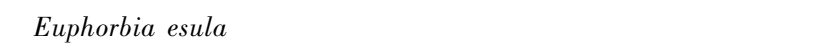

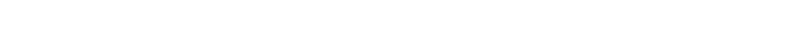

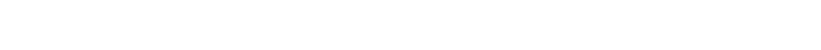

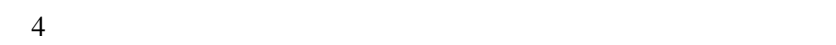

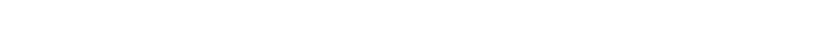

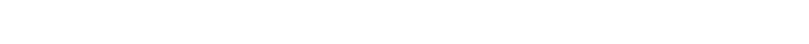

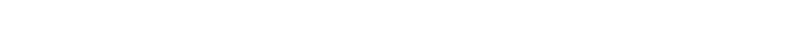

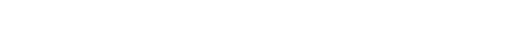

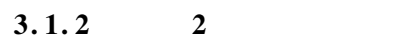

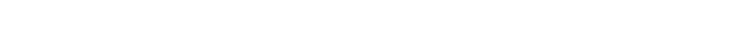

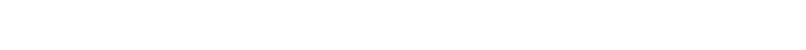

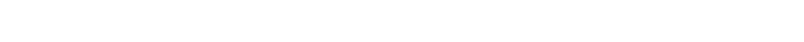

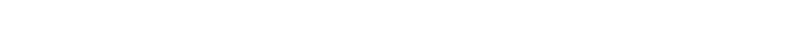

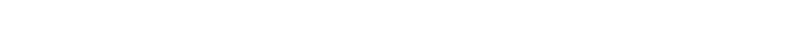

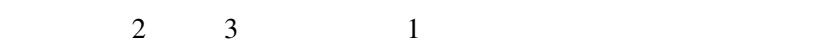

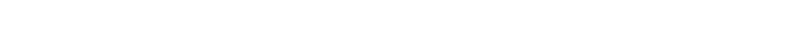

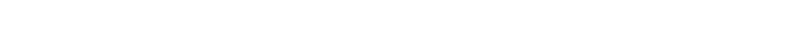

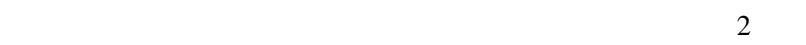

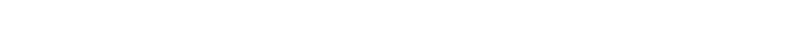

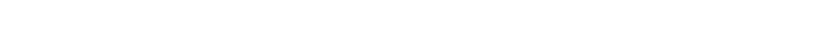

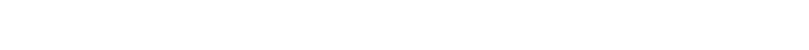

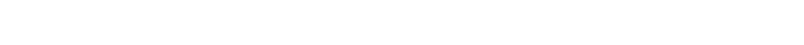

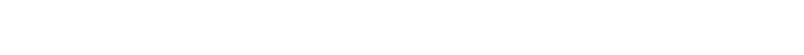

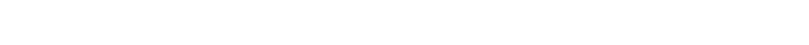

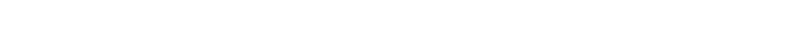

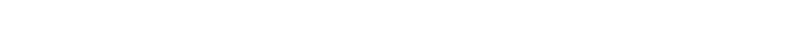

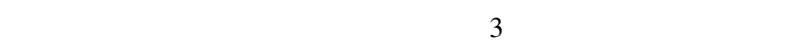

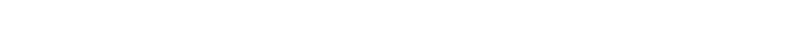

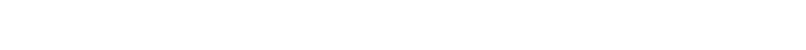

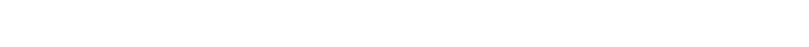

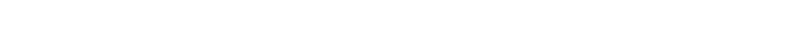

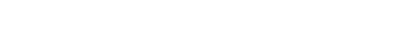

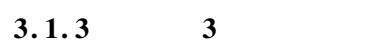

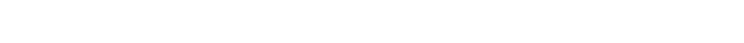

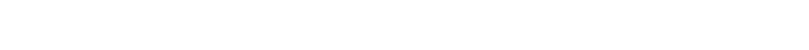

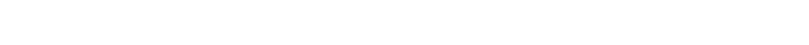

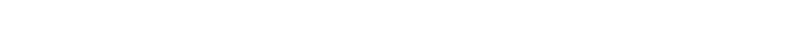

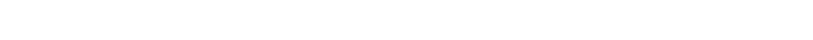

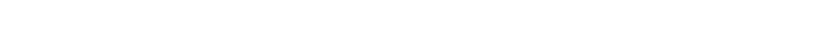

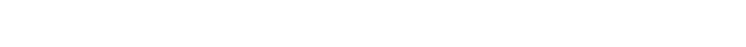

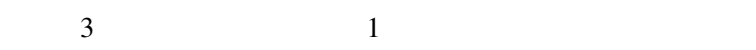

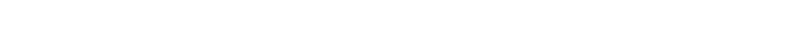

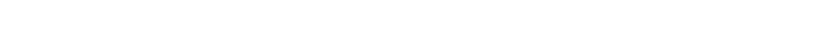

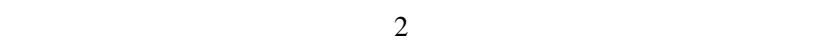

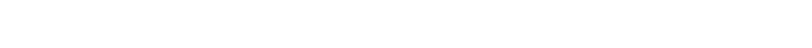

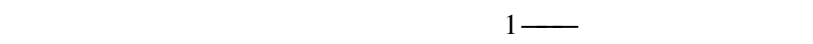

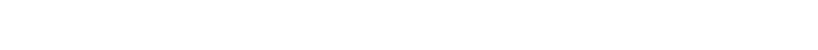

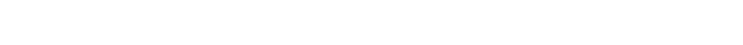

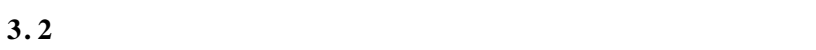
»úö

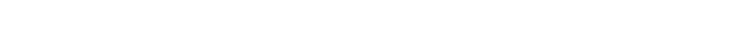

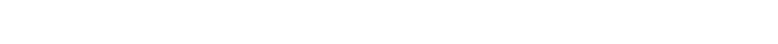

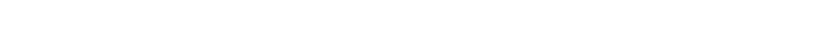

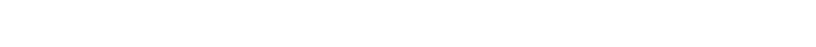

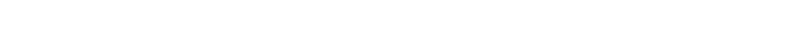
Nuß

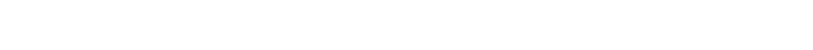

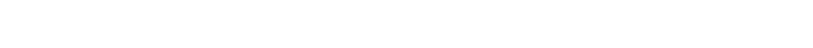

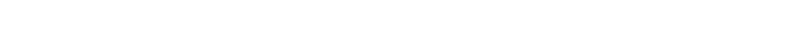

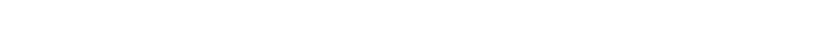
of · p' ÓŎẽ

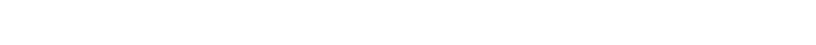

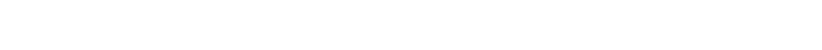

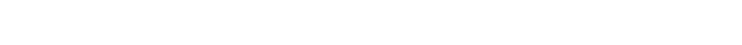

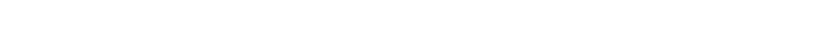

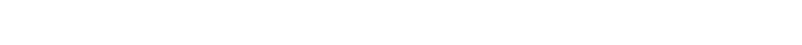

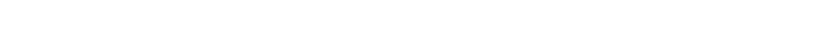

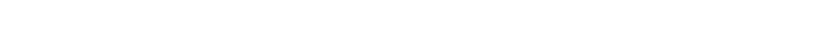

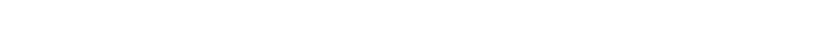

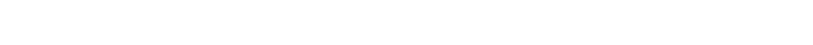

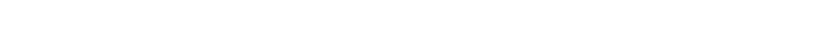

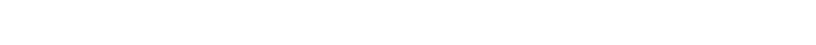

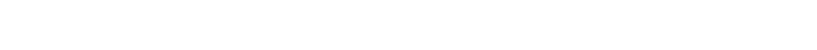

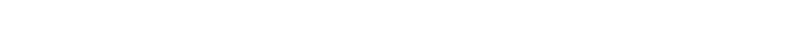

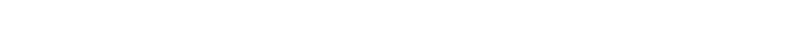
1/A/Ẽ̃̃

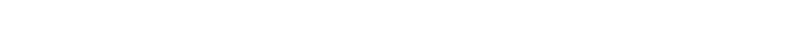

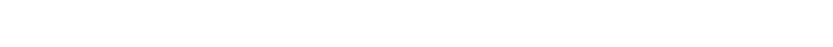

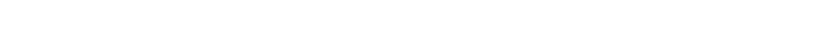

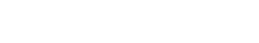

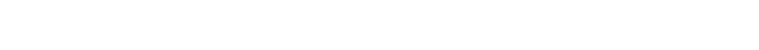

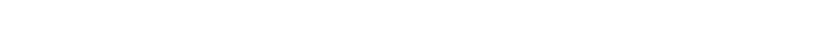

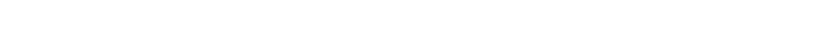




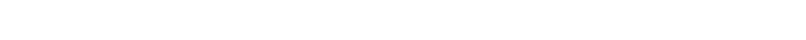

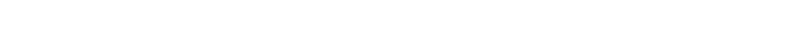
ÖÖळ. Ài

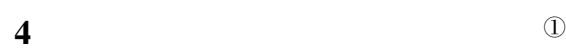

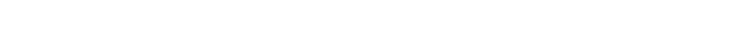

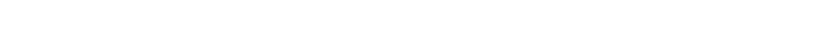

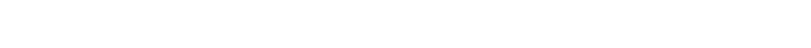

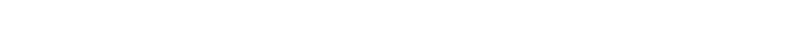

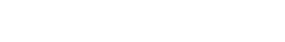

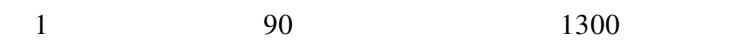

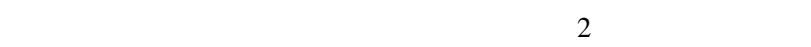

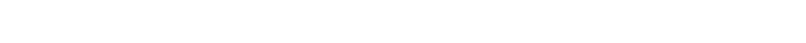

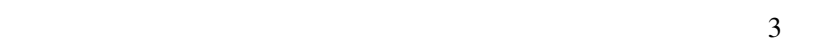

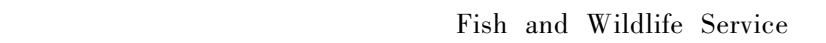

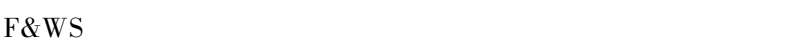

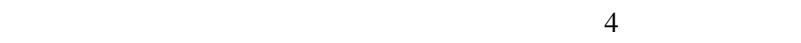

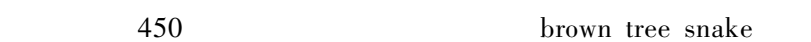

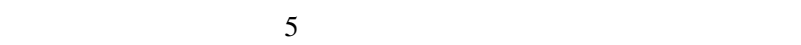

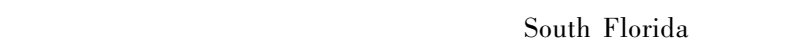

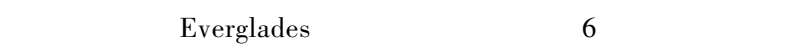

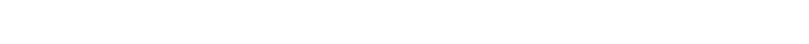

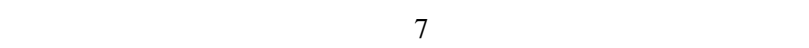

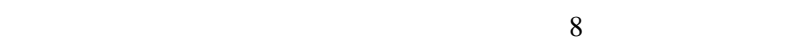

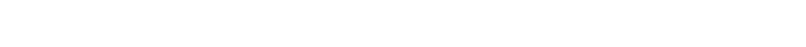

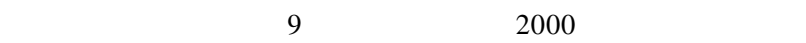

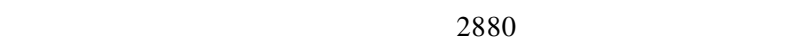

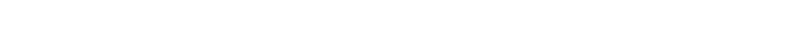

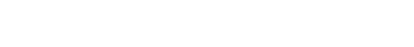

${ }^{2} \hat{\imath} \iota^{1} \hat{\wedge} \hat{4} \ddot{I} x$

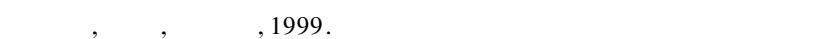

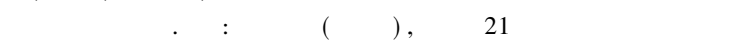

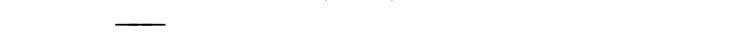

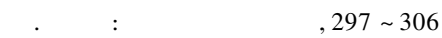

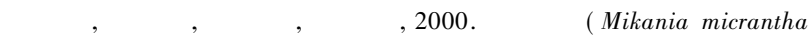

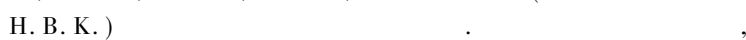
$8(2): 128 \sim 130$

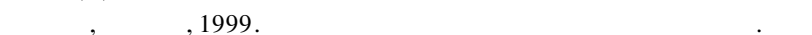
I - $\tilde{N} \pm \ddot{B}, 19(4): 560 \sim 569$

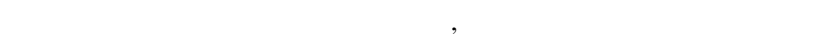

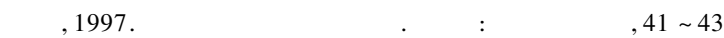

Campbell F T, 1997. Exotic pest plant councils: cooperation to assess and control invasive nonindigenous plant species. In: Luken J O, Thieret J W (eds.) : Assessment and management of plant invasions, $228 \sim 240$

Carlton J T and Geller J B, 1993. Ecological roulette - The global transport of nonindigenous marine organisms. Science, 261: $78 \sim 82$

Hughes F, Vitousek P M and Tunison T, 1991. Alien grass and fire in the seasonal submontane zone of Hawaii. Ecology, 72(2) : $743 \sim 746$

Levine J M, 2000. Species diversity and biological invasions: relating local process to community pattern. Science, 288: $852 \sim 854$

Martin Enserink, 1999. PREDICTING INVASIONS: Biological invaders sweep in. Science, 285: $1834 \sim 1836$

Nichols F H, Thompson J K and Schemel L E, 1990. Remarkable invasion of San Francisco bay (California, USA) by the Asian clam potamocorbula-amurensis. 2. Displacement of a former community. Marine Ecology(Progress Series), 66: $95 \sim 101$

Travis J, 1993. Environmental science ${ }^{a}{ }^{a}$ invader threatens black, Azov Seas. Science, 262: $1366 \sim 1367$

Stachowicz J J, Whitlatch R B and Osman R W, 1999. Species diversity and invasion resistance in a marine ecosystem. Science, 286:1577 1579

U.S. Government Printing Office, 1998. Federal Interagency Committee for the Management of Noxious and Exotic Weeds( eds). "Pulling Together: A National Strategy for Management of Invasive Plants." 2 nd edition. 22 pages.

Wilcove D S, Rothstein D, Dubow J, Phillips A, Losos E, 1998. Quantifying threats to imperiled species in the United States. Bioscience, 48: (8) $607 \sim 615$

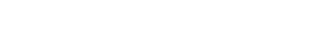

